\title{
THE PYTHAGOREAN GROUP AND ERGODIC FLOWS
}

\author{
BY L. W. GREEN ${ }^{1}$
}

Communicated by G. Hedlund, July 19, 1965

1. Introduction. We outline here a proof of the following

Theorem 1. Let $M$ be a compact $C^{\infty}$ Riemannian manifold, all of whose sectional curvatures are negative. Then the geodesic flow in the orthonormal frame bundle of $M$ is weakly mixing.

J. G. SinaI and D. V. Anosov have proved that the geodesic flow in the tangent bundle induces a Kolmogorov system; in particular, the flow is strongly mixing and has countable Lebesgue spectrum [1], [8]. Indeed, their methods apply to many dynamical systems other than geodesic flows, and Anosov's notion of $U$-system seems to give remarkably complete results in the instability theory of flows and cascades on differentiable manifolds. The extension of a weaker result (weak mixing) from the tangent to the principal bundle would hardly be worthwhile if it were not for the fact that our method seems to provide a new and powerful way of attacking global problems on manifolds with complete connections. Namely, noticing that a welldefined infinite dimensional Lie group (called the Pythagorean group, below) acts transitively on the principal bundle, we try to apply group representation ideas to its analysis.

As in Anosov's proof, one can trace the evolution of our approach back to the Hedlund-Hopf technique of asymptotic geodesics. However, once it is realized that Hopf's introduction of horocyclic coordinates is, in the constant curvature case, a reflection of the Iwasawa decomposition of the associated group, a great many points in his fundamental paper [4] take on new significance. In the Lie group case, this approach has been exploited by L. Auslander, Hahn, and the present author [2], [3], and C. C. Moore [7]. (See also the earlier work by Gelfand and Fomin, and Mautner; references may be found in the above cited papers and book.)

Because we use horocycle flows extensively, a result inaccessible to entropy theory does fall out.

TheOREM 2. Let $M$ be as in Theorem 1 and in addition have dimension two. Then the horocycle flow in the tangent bundle is ergodic.

As far as we know, this is the first such result for a nonmeasure

${ }^{1}$ Research supported by NSF Grant GP-3750. 
preserving flow defined by geometric conditions. We have not yet been able to extend this to higher dimensional manifolds, although it is undoubtedly true without the restriction to surfaces. The details of the proofs will appear elsewhere.

2. The generalized Mautner lemma. Let $V$ be a compact $C^{\infty}$ oriented manifold with a volume element (nonsingular form of highest degree). Then one can talk of the divergence of vector fields on $V$ and of $L_{p}$ spaces of functions on $V$. Thanks to the compactness of $V$, with every vector field $X$ we may associate a one-parameter group of diffeomorphisms, $\left\{X_{t} \mid-\infty<t<\infty\right\}$. Considered as a group of operators in $L_{p},\left\{X_{t}\right\}$ has a closed infinitesimal generator, which we designate also by $X$, since it coincides with the vector field on the $C^{\infty}$ functions. The group $\left\{X_{t}\right\}$ is measure preserving if and only if $\operatorname{div} X=0$. In the spirit of Appendix II of [3], we state the generalized Mautner lemma ${ }^{2}$ as follows:

Proposition 1. Let $X, H$ be infinitesimal generators of one-parameter groups of diffeomorphisms of $V$. Assume $\operatorname{div} X=0$, and that $[X, H]$ $=\lambda H$, where $\lambda$ is a strictly positive function. Then

$$
\lim _{t \rightarrow \infty} X_{-t} H_{8} X_{t}=I
$$

strongly in $L_{2}$ for each $s$. In particular, if $f$ is an eigenfunction of the flow $\left\{X_{t}\right\}$, then $H_{s} f=f$ for all $s$.

The proof of this proposition, so easy when $\lambda$ is constant and $\operatorname{div} H$ $=0$, now uses some formulas from functional analysis. For the $n$ dimensional case we need the analogous result for the case when $H$ is vector valued and $\lambda$ is a definite matrix.

3. The Pythagorean group. Now let $M$ be an oriented, compact $C^{\infty}$ Riemannian manifold of dimension $n$. Let $O(M)$ be the principal bundle of orthonormal frames on $M$. (We use, with discrepancies, the notation and nomenclature of Nomizu-Kobayashi [6].) If $\left\{E_{j}^{i}\right\}, 1 \leqq i<j \leqq n$, is a basis for the Lie algebra of $S O(n)$, there exist vertical vector fields on $O(M)$, which we designate by the same letters, induced by the action of the group on the fibers; these are called fundamental vector fields. Basic vector fields in $O(M)$ are the unique horizontal lifts of geodesics. There exist $n$ independent basic vector fields $B_{1}, \cdots, B_{n}$ such that

$$
\left[B_{i}, E_{k}^{l}\right]=\delta_{k i} B_{l}-\delta_{i l} B_{k}
$$

${ }^{2}$ In this form, the result should be attributed to Segal and von Neumann [9]. 
and

$$
\left[B_{i}, B_{j}\right]=2 \sum R_{m i j}^{r} E_{r}^{m} .
$$

These are the vector forms of the structure equations of $M$ (to which, of course, one adds the Lie algebra multiplication table for the $E^{\prime}$ s).

Since $O(M)$ is complete, each of these $n+\frac{1}{2} n(n-1)$ vector fields gives rise to a one-parameter group of diffeomorphisms. The subgroup generated by these in the full group of diffeomorphisms of $O(M)$ we call the Pythagorean group of $M, P$. The name is chosen because "structure group" means something else, and because relations in this group are consequences, at least locally, of the law of cosines.

The usefulness of $P$ is a consequence of the following properties. First, it acts pointwise transitively in $O(M)$. Of course, except when $M$ is homogeneous, $P$ is not a finite dimensional Lie group, so $M$ is not a coset space of $P$ when any natural topology is assigned to $P$. Next, every element of $P$ preserves the natural measure on $O(M)$ obtained locally from the product of the volume element of $M$ and Haar measure on the fibers (Liouville theorem). Finally, $P$ is finitely generated, at least in the sense that a finite number of one-parameter subgroups generate it, and the relations between their infinitesimal generators are known. Actually, in view of the transitivity of the orthogonal group on the fiber, it is sufficient to take as generators the fundamental vector fields and a single basic one, say $B_{n}$. The oneparameter group of diffeomorphisms generated in $O(M)$ by $B_{n}$ will be called the geodesic flow in $O(M)$.

Proposition 2. Let $f \in L_{2}(O(M))$, and suppose that $f$ is invariant under $P$. Then $f$ is constant.

This proposition is the key to doing ergodic theory on bundles associated with $M$. For example, Theorem 1 is proved by showing that any eigenfunction of the geodesic flow is necessarily invariant under $P$.

A system of root vector fields for the vector field $X$ is defined to be a set $Y_{1}, \cdots, Y_{m}$ of vector fields such that $\left[X, Y_{i}\right]=\sum_{k=1}^{m} \lambda_{i k} Y_{k}$ for some matrix ("root-form matrix") $\left(\lambda_{i k}\right)$. If $m=1, Y$ is simply called a root vector field and $\lambda$ a root function.

Proposition 3. If $M$ has strictly negative sectional curvatures, sufficiently many systems of root vector fields for $B_{n}$ may be found with definite root-form matrices.

Here "sufficiently many" means that the fields in question generate $P$. 
The two-dimensional case is very instructive, although the fact that the tangent bundle and the principal bundle can be identified makes for certain oversimplifications. Let $X$ be the basic vector field inducing the geodesic flow and let $A$ be the unique (within normalization and orientation) fundamental vector field. Another basic field $Y$ can be chosen so the structure equations become

$$
[Y, A]=X, \quad[X, A]=-Y, \quad[X, Y]=k A,
$$

where $k$ is the Gaussian curvature. In analogy with the situation in the Lie algebra of $S L(2, R)$, we seek an eigenvector for ad $X$. One of them is given by

$$
H=Y+u A,
$$

where $u$ is a function on the tangent bundle satisfying the Riccati equation

$$
X(u)+u^{2}+k=0 .
$$

Then $[X, H]=-u H$, and Proposition 1 applies, provided we can obtain estimates for (not to mention, define) $u$. Most of the necessary work in this case has been done by E. Hopf [4], [5]. H, of course, is the infinitesimal generator of the horocycle flow. We see from its definition that the horocycle flow is not measure preserving unless $A(u)=0$. Thus solving the Riccati equation associated with the structure equations (or, if you prefer, with the second variation of arc length) is an essential step in putting the Lie algebra of $P$ into rootspace form. Unfortunately, this process takes us outside of $P$.

Proposition 1 now applies to prove that any eigenfunction of the geodesic flow is invariant under the horocycle flow. Now there are actually two independent horocycle fields, $\mathrm{H}^{+}$and $\mathrm{H}^{-}$, corresponding to the positive and negative asymptotes to geodesics; this is analogous to the presence of positive and negative roots in $\operatorname{sl}(2, R)$. These, in turn, generate $P$, so that Proposition 2 may be invoked. This fact, that $H^{+}$and $H^{-}$generate $P$, is clearly the function analytic counterpart to Sinar's result that the intersection of corresponding $\sigma$-algebras is trivial (cf. the last paragraph of [8]).

The ergodicity of the horocycle flows may be heuristically obtained from the formal relation

$$
X=(A H)^{*}-A H .
$$

Then (still formally; the domains of validity give quite a bit of trouble), if $H f=0$ 


$$
\begin{aligned}
\|X f\|^{2} & =(X f, X f)=\left((A H)^{*} f, X f\right)=(f, A H X f) \\
& =(f, A(u H+X H) f)=0 .
\end{aligned}
$$

Thus any function invariant under the horocycle flow is invariant under the geodesic flow and therefore constant. ${ }^{3}$

4. Remarks. Anosov has pointed out that, in a general $U$-system, it is naive to hope for the existence of transverse fields such as our horocycle flows. But, as we remarked above, it would be silly to attempt to replace the methods of entropy theory when they yield such strong conclusions. It is in the borderline area, where structural stability probably does not hold, that there is the most promise for Lie algebra techniques. In this connection, we remark that our work shows the usefulness of weaker concepts of root and Cartan subalgebra than promulgated by, say, Sternberg. ${ }^{4}$

Perhaps more appropriate uses of the Pythagorean group are in global differential geometry. For instance, although the proof is essentially the usual one, de Rham's decomposition theorem becomes conceptually more satisfying when phrased in terms of complete reducibility of the Pythagorean group. In general, one can now formulate many questions which previously made sense only in symmetric spaces, for arbitrary complete Riemannian manifolds.

Added in Proof. Sinar has called to my attention that the result of Theorem 2 is essentially contained in the course of his proof that geodesic flows on surfaces are $K$-systems (Geodesic flows on compact surfaces of negative curvature, Dokl. Akad. Nauk SSSR 136 (1961), 549-552 =Soviet Math. Dokl. 2 (1960), 752-758).

\section{REFERENCES}

1. D. V. Anosov, Ergodic properties of geodesic flows, Dokl. Akad. Nauk SSSR 151 (1963), 1250-1252=Soviet Math. Dokl. 4 (1963), 1153-1155.

2. L. Auslander, L. W. Green, and F. Hahn, Flows on homogeneous spaces, Annals of Mathematics Studies, No. 53, Princeton University Press, Princeton, N. J., 1963.

3. L. Auslander and L. W. Green, G-induced flows, Amer. J. Math. (to appear).

4. E. Hopf, Statistik der geodätischen Linien in Mannigfaltigkeiten negativer Krümmung, Ber. Verh. Sächs. Akad. Wiss. Leipzig 91 (1939), 261-304.

5. - Statistik der Lösungen geodätischer Probleme von unstabilen Typus. II, Math. Ann. 117 (1940), 590-608.

${ }^{8}$ This particular approach is new even in the constant curvature case. T. Sherman in [10] uses a similar argument on the triple $X, H^{+}$, and $H^{-}$to examine representations of $S L(2, R)$.

4 We remark further that, unless $M$ has dimension two, the flow in the principal bundle is not a $U$-system. However, the method of transverse fields undoubtedly would show that it is a $K$-flow. (Cf. Sinar's Stockholm Congress report, 1962. Our method exposes the "fine structure" responsible for the triviality of his $\mathfrak{R}_{Z}$.) 
6. S. Kobayashi and K. Nomizu, Foundations of differential geometry, Interscience, New York, 1963.

7. C. C. Moore, Ergodicity of flows on homogeneous spaces, (to appear).

8. J. G. Sinal, Proof of the ergodic hypothesis for a dynamical system of statistical mechanics, Dokl. Akad. Nauk SSSR 153 (1963), 1261-1264=Soviet Math. Dokl. 4 (1963), 1818-1822.

9. I. Segal and J. von Neumann, $A$ theorem on unitary representations of semisimple Lie groups, Ann. of Math. 52 (1950), 509-517.

10. T. Sherman, $A$ weight theory for unitary representations, (to appear).

UNIVERSITY OF MINNESOTA

\section{$L_{p q}$ INTERPOLATORS AND THE THEOREM OF MARCINKIEWICZ}

BY EVELIO T. OKLANDER

Communicated by A. Zygmund, July 27, 1965

The purpose of this paper is to introduce certain interpolation methods (interpolators) which lead to a proof of Marcinkiewicz's theorem. We start with some definitions.

An interpolation pair is a couple of Banach spaces continuously contained in a Hausdorff topological vector space $V$.

On the vector spaces $A_{1}+A_{2}=\left\{u \in V: u=v+w, v \in A_{1}, w \in A_{2}\right\}$ and $A_{1} \cap A_{2}$ we introduce the norms

$$
\begin{aligned}
& \|u\|_{A_{1}+A_{2}}=\inf \left\{\|v\|_{A_{1}}+\|w\|_{A_{2}}: v+w=u, v \in A_{1}, w \in A_{2}\right\}, \\
& \|u\|_{A_{1} \cap A_{2}}=\max \left\{\|u\|_{A_{1}},\|u\|_{A_{2}}\right\}
\end{aligned}
$$

with these norms, $A_{1}+A_{2}$ and $A_{1} \cap A_{2}$ become Banach spaces.

An interpolator $F$ is a function defined on interpolation pairs whose values are Banach spaces $F\left(A_{1}, A_{2}\right)$ such that:

(1) $A_{1} \cap A_{2} \subset F\left(A_{1}, A_{2}\right) \subset A_{1}+A_{2}$, the inclusions being continuous;

(2) if $\left(X_{1}, X_{2}\right),\left(Y_{1}, Y_{2}\right)$ are interpolation pairs, and $T$ is a linear map of $X_{1}+X_{2}$ into $Y_{1}+Y_{2}$ which maps $X_{1}$ into $Y_{1}$ and $X_{2}$ into $Y_{2}$ and which decreases the norms, then $T$ is also a norm decreasing map of $F\left(X_{1}, X_{2}\right)$ into $F\left(Y_{1}, Y_{2}\right)$.

We will say that $F\left(A_{1}, A_{2}\right)$ is an intermediate space between $A_{1}$ and $A_{2}$.

The functions considered in the following are complex-valued functions defined on a totally $\sigma$-finite measure space $(M, m)$. The distribution function of $f$ is 